\title{
Green and efficient inkjet printing of cotton fabrics using reactive dye@copolymer nanospheres
}

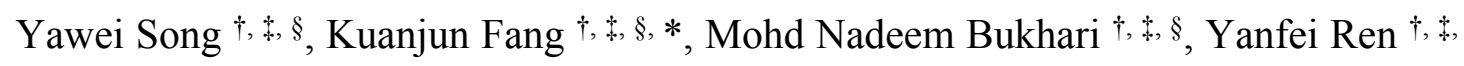

$\S$, Kun Zhang ${ }^{\dagger}, \ddagger, \S$, Zhiyuan Tang $\dagger, \ddagger, \S$

$\dagger$ State Key Laboratory of Bio-Fibers and Eco-Textiles, Qingdao University, 308 Ningxia Road, Qingdao 266071, China.

¥ Collaborative Innovation Center for Eco-Textiles of Shandong Province, 308 Ningxia Road, Qingdao 266071, China.

$\S$ School of Textiles \& Clothing, Qingdao University, 308 Ningxia Road, Qingdao 266071, China.

*Corresponding author: Email: 13808980221@163.com

Address: School of Textiles \& Clothing, Qingdao University, 308 Ningxia Road, Qingdao 266071, Shandong, China. 

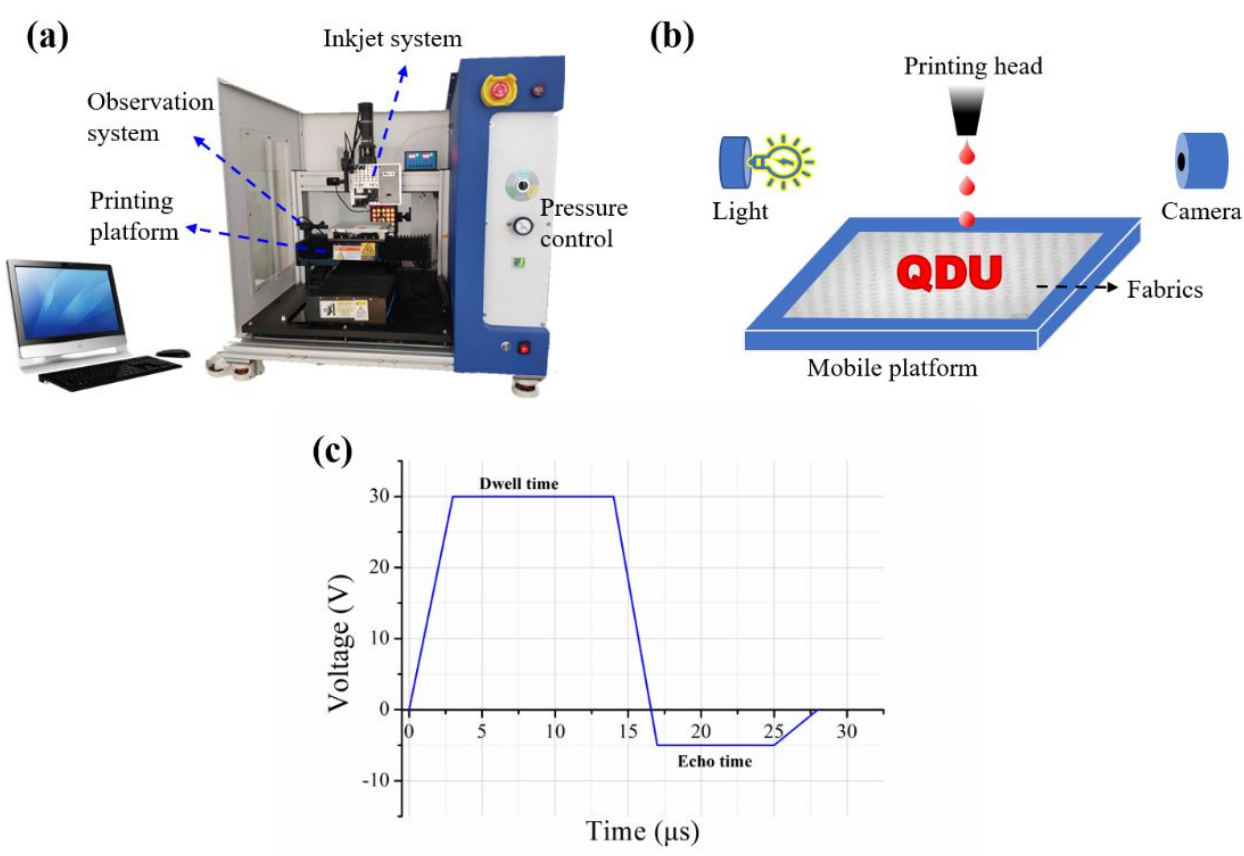

Figure S1. (a) The inkjet printing machine. (b) Schematic diagram of droplet observation and inkjet printing system. (c) The bipolar control waveform used for droplet generation. The experiments were conducted at $25^{\circ} \mathrm{C}$ with a relative humidity is $70 \%$. Nozzle diameter was $40 \mu \mathrm{m}$. Air pressure was controlled at $-1.7 \mathrm{MPa}$. Jetting frequency was $1000 \mathrm{~Hz}$. 


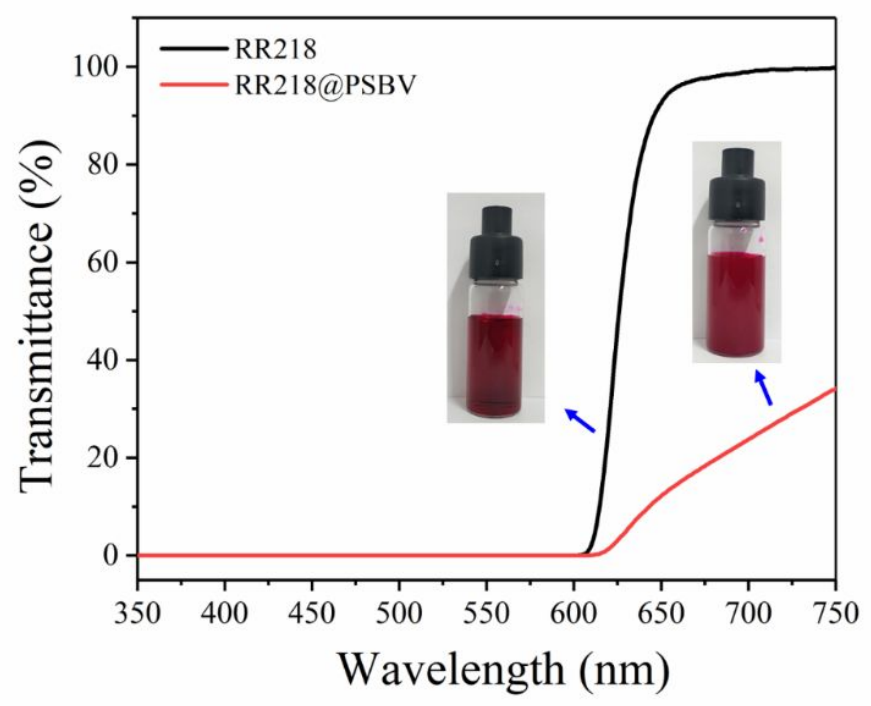

Figure S2. The transmittance of RR218 solution and RR218@PSBV dispersion. The concentration of RR218 solution was 1 mmol/L, and the RR218@PSBV dispersion was $8.3 \mathrm{~g} / \mathrm{L}$ with same amount of RR218 dyes. 
(a)

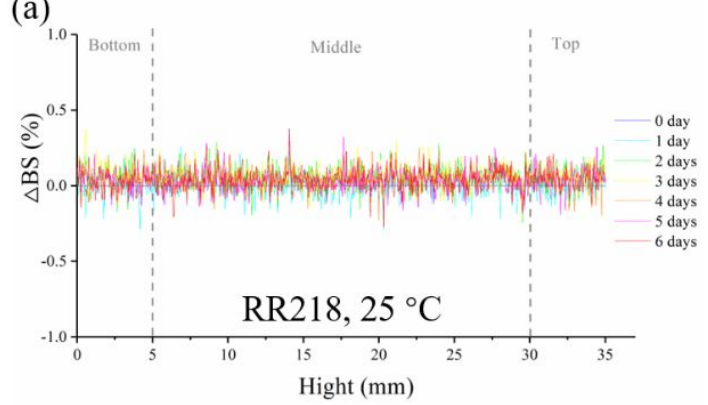

(b)

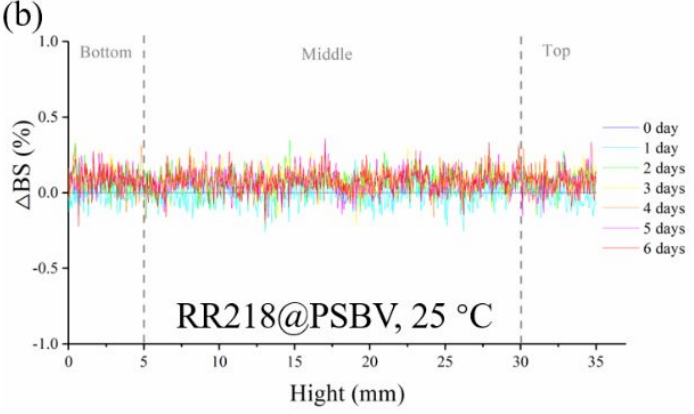

Figure S3. Backscattering profiles vs. sample height of RR218 solution (a) and RR218@PSBV dispersion (b). The concentration of RR218 solution was $1 \mathrm{mmol} / \mathrm{L}$, and the RR218@PSBV dispersion was $8.3 \mathrm{~g} / \mathrm{L}$ with same amount of RR218 dyes.

\section{Test principle of Turbiscan Lab :}

The stability was determined by Turbiscan Lab, which is able to measure the various dispersion states by multiple light scattering. The Turbiscan Lab was used to detect and characterize various concentrated dispersions destabilization (coalescence, flocculation, creaming and sedimentation) ${ }^{35-37}$. Typically, the test sample is placed in a glass cylinder sample bottle. Then the incident light with an $880 \mathrm{~nm}$ wavelength will scan the sample bottle periodically from the bottom to top. For particle dispersions, the intensity of backscattering (BS) light will be measured at $135^{\circ}$ from the incident beam. Based on Mie theory, the intensity of backscattering (BS) of incident light can be expressed through the calculation of the transport mean free path of the photons $\left(l^{*}\right)$ :

$$
\begin{gathered}
B S \approx\left(\frac{l}{l^{*}}\right)^{1 / 2} \\
l^{*} \approx \frac{2 d}{3 \varphi(1-a) Q_{s}} \\
l=\frac{2 d}{3 \varphi Q_{s}}
\end{gathered}
$$


where $d, \varphi, h$, and $Q_{s}$ represent the diameter of particles, the volume fraction of dispersed phase, asymmetry factor, and scattering efficiency factor, respectively.

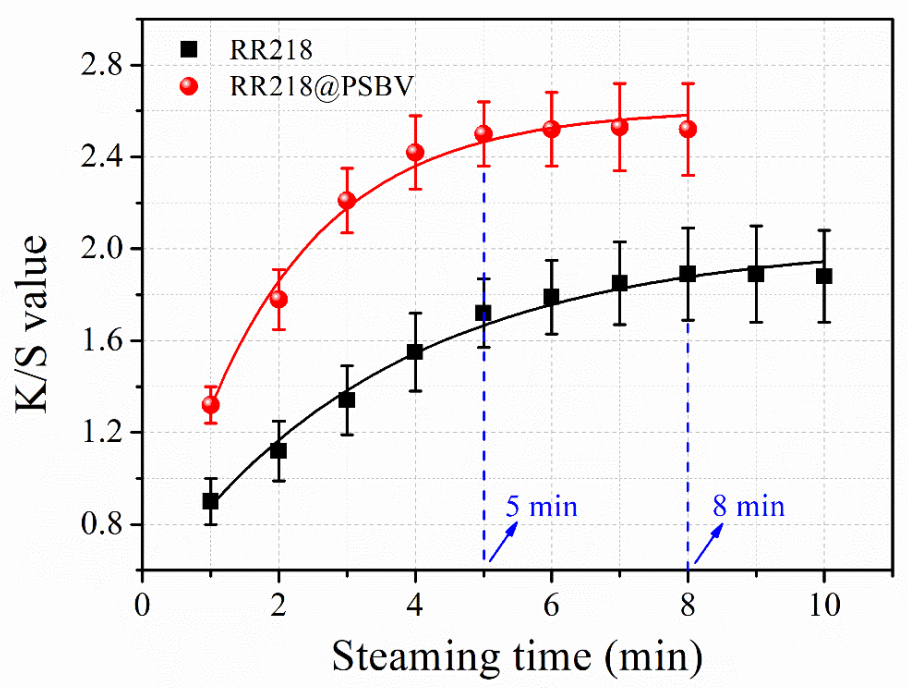

Figure S4. The effect of steaming time on $K / S$ values of printed fabrics. The concentration of RR218 solution was $1.62 \mathrm{mmol} / \mathrm{L}$, and the RR218@PSBV dispersion was $13.6 \mathrm{~g} / \mathrm{L}$ with same dye amount with RR218 solution. 
Table S1. The fastness rating of RR218 and RR218@PSBV printed patterns.

\begin{tabular}{|c|c|c|c|c|}
\hline \multirow{2}{*}{ Sample } & \multicolumn{2}{|c|}{ Rubbing fastness } & \multicolumn{2}{c|}{ Washing fastness } \\
\cline { 2 - 5 } & dry & wet & change & staining \\
\hline RR218 & 4 & 4 & 4 & $3-4$ \\
\hline RR218@PSBV & 4 & $3-4$ & 4 & $3-4$ \\
\hline
\end{tabular}

a The concentration of RR 218 solution was $1 \mathrm{mmol} / \mathrm{L}$

bRR218@PSBV dispersion was 8.3 g/L with same dye amount of RR218 solution

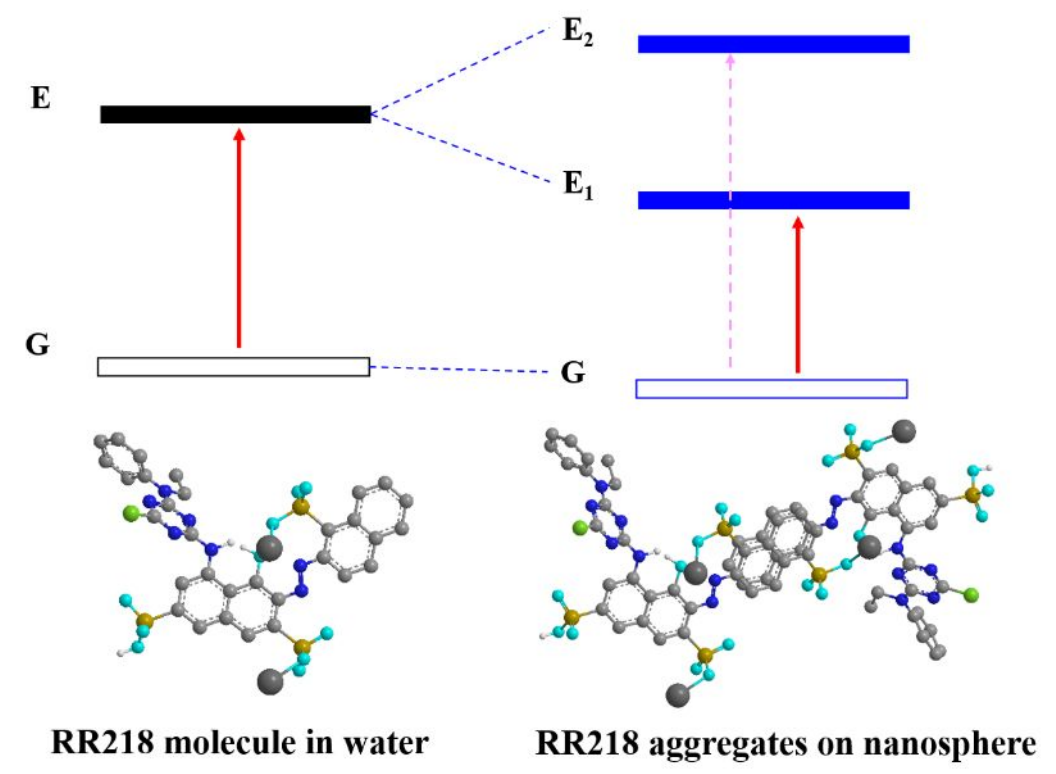

Figure S5. Exciton energy diagram for dye molecules and dye aggregates with linear structure. 

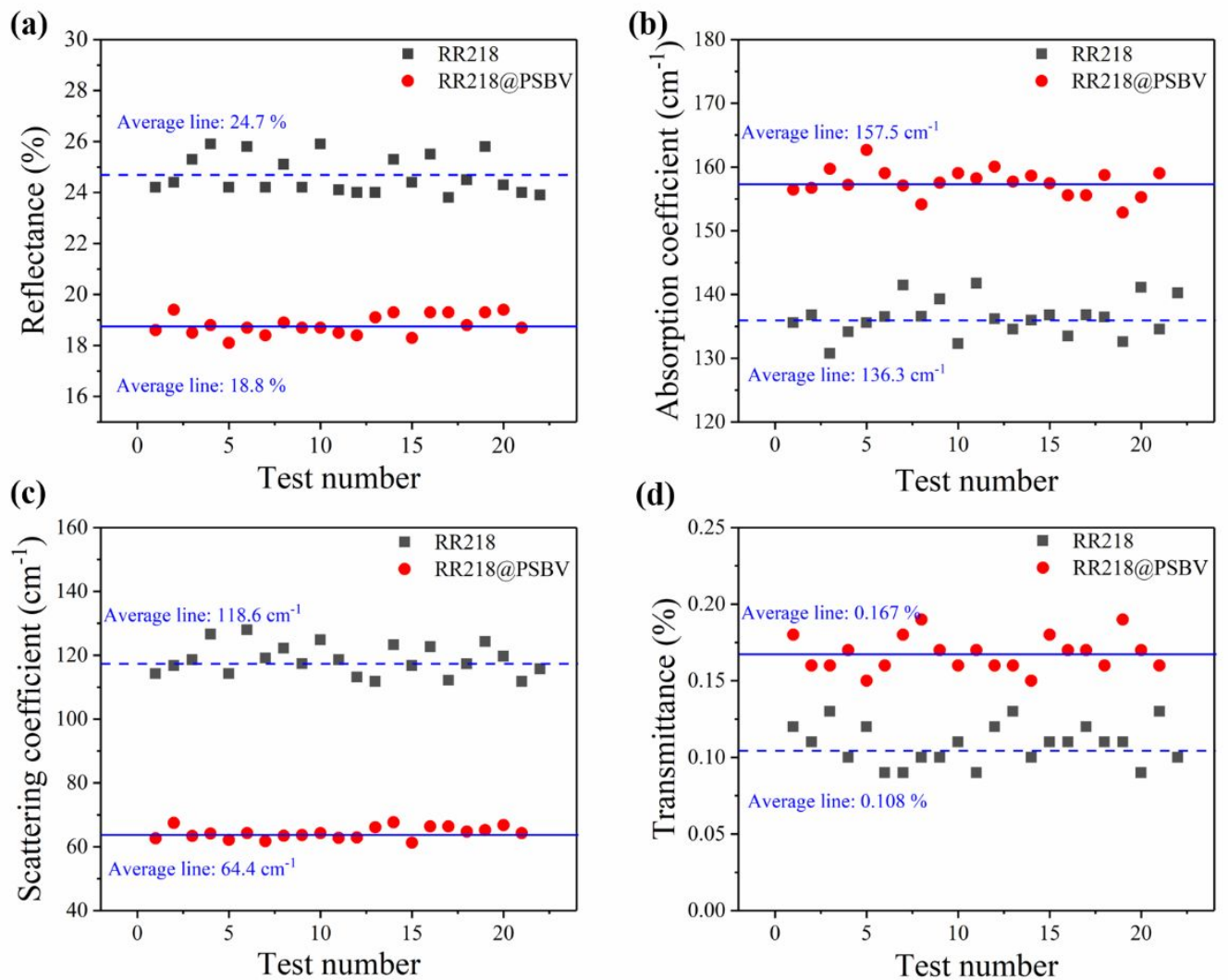

Figure S6. Comparison of the reflectance (a), absorption coefficient (b), scattering coefficient (c) and transmittance (d) at different points of printed fabrics using RR218 and RR218@PSBV. The concentration of RR218 solution was $1 \mathrm{mmol} / \mathrm{L}$, and the RR218@PSBV dispersion was 8.3 g/L with same amount of RR218 dyes. 